Original Article

\title{
AN IMPROVED LC-MS/MS METHOD DEVELOPMENT AND VALIDATION FOR THE DETERMINATION OF TRANDOLAPRIL AND VERAPAMIL IN HUMAN PLASMA
}

\author{
YATHA RAVI*, B. RAJKAMAL
}

${ }^{1}$ Mewar University, Chittorgarh, Rajasthan, India

Email: ravi_ard@rediffmail.com

Received: 02 Dec 2018 Revised and Accepted: 04 Feb 2019

\section{ABSTRACT}

Objective: A simple, sensitive and rapid LC-MS/MS technique was developed for the quantitation of trandolapril (TDL) and verapamil (VPL) in a biological matrix and validated.

Methods: Sample preparation processed by SPE (Solid Phase extraction) on phenomenex cartridge using Ledipasvir as an internal standard. Two drugs were eluted on waters symmetry- $\mathrm{RP}_{18}(5 \mu, 150 \mathrm{~mm} \times 4.0 \mathrm{~mm})$ column with the mobile composition of 10 mmol ammonium formate and $\mathrm{ACN}$ (acetonitrile) in the ratio of 70:30\%V/V. Detection and quantitation were processed by electrospray ionization in positive ionization mode.

Results: The quantification approach was validated in 5-1500 ng/ml linear concentration range for TDL and 1-2000 ng/ml for VPL. The intraday and inter-day precision and accuracy were found to be $0.58 \%$ to $5.69 \%$ and $93 \%$ to $104 \%$ for two drugs. The average recoveries for TDL and VPL were found to be $92.9 \%$ and $93.5 \%$ respectively.

Conclusion: The developed work was validated and can be applicable to the routine analysis of TDL and VPL simultaneously in a biological matrix

Keywords: Trandolapril, Verapamil, Cancer, ACE inhibitor, Accuracy, LC-MS/MS, Validation

(C) 2019 The Authors. Published by Innovare Academic Sciences Pvt Ltd. This is an open access article under the CC BY license (http://creativecommons.org/licenses/by/4.0/) DOI: http://dx.doi.org/10.22159/ijpps.2019v11i3.31247

\section{INTRODUCTION}

TDL is a nonsulfhydryl prodrug that is de-esterified to trandolaprilat in the liver, belongs to the class of an angiotensin-converting enzyme (ACE) inhibitor medications [1]. It obstructs ACE, which shows a significant role in the conversion of angiotensin-I (AT-I) to angiotensin-II (AT-II). AT-II controls blood pressure and is a main constituent of the RASS (renin-angiotensin aldosterone system) [2]. It helpful in hypertension treatment and increase survival subsequent myocardial infarction patients suffering from left ventricular dysfunction. It acts as an adjunct in heart failure and reduces the rate of progress of the renal disease in patients with hypertension, diabetes and overt nephropathy. It chemically designated as (2S, 3aR, 7aS)-1-[(2S)-2-\{[(2S)-1-ethoxy-1-oxo-4phenylbutan-2-yl] amino\} propanol]-octahydro-1H-indole-2carboxylic acid $[3,4]$.

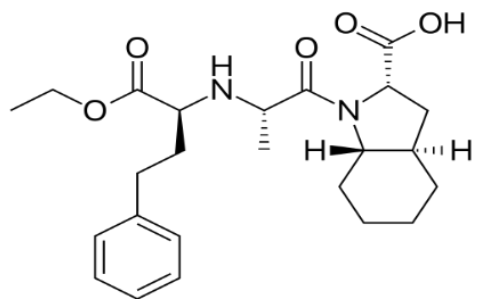

Fig. 1: Structure of trandolapril

VPL is useful in the treatment of high blood pressure by dilating the blood vessels, angina by increasing the oxygen and blood supply to the heart and supraventricular tachycardia. It also useful in the prevention of a migraine and cluster headaches. The drug was administered either by oral route or through injection into a vein. It acts by blocking the voltage-dependent calcium channel and this drug considered as a class-IV antiarrhythmic agent in cardiac pharmacology $[4,5]$. IUPAC name of the compound was $(R S)-2-(3,4-$
Dimethoxyphenyl)-5-\{[2-(3, 4-dimethoxyphenyl) ethyl]-(methyl) amino\}-2-prop-2-yl pentanenitrile [4, 6]. Chest pain is controlled by using verapamil regularly.

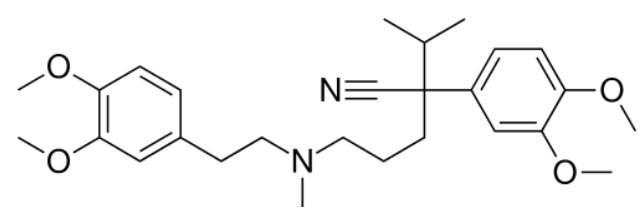

Fig. 2: Structure of verapamil

Literature survey of TDL and VPL revealed that several analytical techniques were employed such as high-performance thin layer chromatography (HPTLC) [7], high-performance liquid chromatography (HPLC) [8-12] and LC-MS/MS [13, 14]. Aim of the present work was to develop an improved, accurate and rapid LC-MS/MS technique with low retention time.

\section{MATERIALS AND METHODS}

\section{Chemicals and reagents}

The TDL, VPL reference standards and internal standard (Ledipasvir) were obtained from Hetero drugs, Hyderabad. HPLCgrade ACN and methanol were procured form SD-Fine Chemicals, Mumbai, India. Phenomenex SPE-cartridges were bought from Phenomenex, Bengaluru, India. Analytical grade ammonium formate and formic acid were bought from Qualigens chemicals, Mumbai, India.

\section{Mass spectrometric and liquid chromatographic conditions}

Liquid chromatographic system (Shimadzu, Japan) consisted of a binary LC-20A CE pump, solvent degasifying system (DGU-20A), autosampler (SIL-HTC) and temperature controller (CTO-10 AS) for maintaining column temperature was used for the chromatographic elution of the two drugs and internal standard (IS). Separation of 
analyte peaks was carried out with Waters symmetry-RP 18 (150 $\mathrm{mm} \times 4.0 \mathrm{~mm}, 5 \mu$ ) column at $40^{\circ} \mathrm{C}$ oven temperature with the mobile composition of ACN and $10 \mathrm{mmol}$ ammonium formate in the ratio of $70: 30 \mathrm{v} / \mathrm{v}$. The mobile phase flow of $0.9 \mathrm{ml} / \mathrm{min}$ was processed through an analytical column with $5{ }^{\circ} \mathrm{C}$ autosampler temperature.

Mass spectrometric detection was processed using API-4000 mass spectrometer (MDS-SCIEX, Toronto, Canada) combined with ESI (electrospray ionization). The MRM transitions for the quantitation of TDL, VPL and Ledipasvir were $\mathrm{m} / \mathrm{z}$ 431.2/234.1, m/z 455.0/165.0 and $\mathrm{m} / \mathrm{z}$ 889.4/130.0 respectively. The optimized mass parameters like ion-spray voltage, curtain gas, Gas-1, Gas-2, collisional activation dissociation, and turbo heater temperature were processed at 2500 V, 30 psi, 40 psi, 65 psi, 8 psi and $450{ }^{\circ} \mathrm{C}$ respectively. The parameters depends on the compound, namely declustering potential(DP), entrance potential, collision cell exit potential (CEP) and collision energy were adjusted to $90 \mathrm{~V}, 9 \mathrm{~V}, 12 \mathrm{~V}$ and $25 \mathrm{~V}$ for TDL and $80 \mathrm{~V}, 9 \mathrm{~V}, 10 \mathrm{~V}$ and $25 \mathrm{~V}$ for VPL respectively.

\section{Quality and calibration control samples}

TDL and VPL stock solutions ( $200 \mu \mathrm{g} / \mathrm{ml})$ were freshly processed by dissolving accurate quantities of drugs in methanol as solvent separately. The quality control (QC) samples and calibration standards (CS) were processed by spiking with suitable volumes of working standard solutions made from intermediate stocks for the two drugs to blank plasma. The QC concentrations for TDL/VPL were LLOQ (lower limit of quantification): $5 / 1 \mathrm{ng} / \mathrm{ml}$, Low QC (lowquality control): $10 / 2 \mathrm{ng} / \mathrm{ml}$, Medium QC (medium-quality control): 600/600 ng/ml and High QC (high-quality control): 1100/1500 $\mathrm{ng} / \mathrm{ml}$. The CSs were $5,10,150,300,400,600,850,1100$ and 1500 $\mathrm{ng} / \mathrm{ml}$ for TDL and 1, 2, 40, 200, 350, 600, 900, 1500 and 2000 $\mathrm{ng} / \mathrm{ml}$ for VPL. Ledipasvir (LPR) $100 \mu \mathrm{g} / \mathrm{ml}$ stock solution was prepared by dissloving an accurate amount of IS in methanol. LPR 8 $\mu \mathrm{g} / \mathrm{ml}$ working standard was made from $50 \%$ methanol. Standard and stock sample solutions were kept at 2 to $8{ }^{\circ} \mathrm{C}$ and QC standards and CSs were kept at- $70^{\circ} \mathrm{C}$ till actual sample analysis.

\section{Preparation of sample solution}

Prior to the separation, all samples subjected for freezing, QCs and CSs sample concentrations were equilibrated to room temperature. To $250 \mu \mathrm{l}$ of spiked sample, $50 \mu \mathrm{l}$ of LPR was mixed and subjected to vortex for $30 \mathrm{sec}$. Further $150 \mu \mathrm{l}$ of buffer $(10 \mathrm{mmol}$ ammonium formate $\mathrm{pH}$ 3.2) was added and placed in a centrifuge for $15 \mathrm{~min}$ at $4500 \mathrm{rpm}$. Further samples were processed through pre-treated phenomenex cartridges with methanol and $10 \mathrm{mmol}$ ammonium formate buffer. The sample matrix was exhausted out from the cartridges by the application of positive pressure (nitrogen). The cartridges were cleaned with $1 \mathrm{ml}$ of $10 \mathrm{mmol}$ ammonium formate buffer and $1 \mathrm{ml}$ of $50 \%$ methanol. The IS and drugs were separated with $500 \mu \mathrm{l}$ of the mobile phase into pre-labeled vials.

\section{System suitability and sensitivity}

System suitability experimentation was executed by introducing six repeated injections, using an extracted sample of TDL/VPL and internal standard at the starting of each lot. Analytical technique selectivity was analysed for potential interfering matrix components in $10 \mathrm{dfferent}$ sources (6 Na-heparinized, 2-haemolysed and 2-lipemic) of blank plasma sample by extraction and examination of the resultant chromatograms for interfering matrix components peaks [15-18]. Analytes stock solutions were processed by dissolving required quantity in methanol. Working solutions were processed in $50 \%$ methanol and 5 $\mu \mathrm{l}$ was introduced in to the system to check for possible interfering constituents at the elution time of the drugs and IS.

\section{Accuracy and precision}

Intra-day accuracy and precision were evaluated by identical analysis of samples on the same day. The sample runs consist of calibration standards and 6 replicates of LLOQ Lower-QC, Medium-QC, and High-QC samples. The inter-day accuracy and precision were evaluated by determination of 3 accuracy and precision lots on three di fferent days [19-21]. The \% CV at each sample concentration should be $<15 \%$. Similarly, an average accuracy should be between $85 \%-115 \%$ all and for LLOQ it should be $80 \%-120 \%$ of the nominal concentration.

\section{Linearity and re-injection reproducibility}

The five calibration curve lines were plotted by preparing and analysing 9 different concentration solutions for the estimation of linearity. Linear $\left(1 / x^{2}\right)$, least squares regression algorithm was used to construct linearity plot from the data of peak response ratio (analyte/IS) versus concentration. The linearity formula was used to estimate the projected concentrations in samples within the analytical runs. The correlation coefficient of calibration curves should to be more than 0.99 for the two analytes [16]. Re-injection reproducibility estimated for extraction samples by re-injecting the sample after storing at $5^{\circ} \mathrm{C}$.

\section{Method ruggedness}

To find out method ruggedness of analytes, two lots were studied for accuracy and precision. The first lot was assessed on two SymmetryShieldRP $18-(150 \mathrm{~mm} \times 3.9 \mathrm{~mm}, 5 \mu \mathrm{m})$ analytical columns with different batch numbers and the second lot was studied by two different analysts.

\section{Carryover effect}

Carryover effect was assessed to confirm that the washing solution used to clean the infusing needle and the port is able to evade any carry forward of an introduced sample in preceding runs. Autosampler carryover was estimated by consecutively introducing extracted blank plasma, duplicate LLOQ duplicate ULOQ sample followed by introducing duplicate blank plasma. Cross selectivity test was executed to check the conversion of TDL to VPL and vice versa during successive steps of analysis [14]. This test was performed at LLOQ and HQC levels for both the analytes in duplicate and processed along with two blank plasma samples.

\section{Matrix effect and Recovery}

Extraction retrieval of the drugs and IS from biological matrix was assessed in six duplicates by comparing the maverage peak responses of pre-extraction fortfied samples to those of post extraction fortified samples. Absolute matrix effect was evaluated by paralleling the average peak responses of post-extraction fortified samples to those of neat samples prepared in elution solution. To evaluate the relative matrixffect in different plasma lots, post extraction fortified samples were prepared in duplicate at Low-QC and High-QC concentrations and \% CV was assessed. Effect of matrix ions on drugs sensitivity were estimated by analyte infusion [13] Briefly, TDL, VPL and Ledipasvir (at ULOQ level) standard solution was injected post-column through the mobile phase having infusion pump. $5 \mu$ l aliquots of extracted control samples were then infused into the column and anlyte chromatograms were estimated.

\section{Stability assessment}

TDL and VPL stock solutions and IS were tested at room conditions (short-term stability at $25^{\circ} \mathrm{C}$ ) and at $5{ }^{\circ} \mathrm{C}$ (long-term stability). Stability findings were estimated by determining the peak area (analyte/IS) of samples against freshly processed samples with same concentration. The $\%$ difference should be $\pm 10 \%$ for both stability solutions. Freezethaw (at $-70{ }^{\circ} \mathrm{C}$ ) stability, bench-top stability, autosampler (at $5{ }^{\circ} \mathrm{C}$ ) stability, processed sample (at $25^{\circ} \mathrm{C}$ ) stability and long term (at $-70{ }^{\circ} \mathrm{C}$ ) stability were processed at Low-QC and High-QC levels using 6 replicates. The stability solutions were estimated against freshly processed calibration standards [22-24].

\section{Dilution integrity}

It was processed to validate the test for dilution, performed on higher concentrations of analyte (above ULOQ). It was processed at 1.6 times the ULOQ concentration and 6 duplicate samples of $1 / 4$ and $1 / 2$ concentrations were processed. The concentrations were estimated by the application of dilution factor 4 and 2 respectively by comparing with the freshly processed calibration standards $[15,16]$.

\section{RESULTS AND DISCUSSION}

\section{Method development}

Present method was projected to improve upon the existing methods in order to achieve higher sensitivity, lower sample volume requirement for extraction and use of Ledipasvir internal standard 
for better precision and accuracy. Moreover, none of the reported method afforded baseline chromatographic separation of TDL and VPL under the established chromatographic conditions. Both TDL and VPL were processed in the positive mode and the ions of precursor and product were optimized for two drugs and ledipasvir $150 \mathrm{ng} / \mathrm{ml}$ solutions by injecting in mass range of 50-500 Da. The Q1 full scan for two drugs and ledipasvir primarily contains protonated precursor $[\mathrm{M}+\mathrm{H}]^{+}$ions at $\mathrm{m} / \mathrm{m} / \mathrm{z} 431.2,455.0$ and 889.4 for TDL, VPL and IS respectively. The corresponding stable and abundant productions in Q-3 MS were detected at $\mathrm{m} / \mathrm{z} 234.1,165.0$ and 130.0 respectively. The MRM transitions for the quantification of TDL, VPL and IS were $\mathrm{m} / \mathrm{z} 431.2 / 234.1, \mathrm{~m} / \mathrm{z}$ 455.0/165.0 and $\mathrm{m} / \mathrm{z}$ $889.4 / 130.0$ respectively. The chromatographic parameters were optimized to increase peak response and reduce the interference of impurity peaks. In order to achieve this, several reversed-phase columns like Hypersil-Gold ( $5 \mu, 150 \mathrm{~mm} \times 4.6 \mathrm{~mm}$ ), HypurityAdvance $(5 \mu, 150 \mathrm{~mm} \times 4.6 \mathrm{~mm})$, BDS Hypersil-C ${ }_{18}(5 \mu, 150 \mathrm{~mm} \times$ $4.6 \mathrm{~mm})$ and symmetry-RP $18(5 \mu, 150 \mathrm{~mm} \times 4.0 \mathrm{~mm})$ column were verified using similar mobile phase. All four columnsfforded chromatographic elution of the analytes but the response was not adequate especially at the LLOQ levels of TDL and VPL. However, the signal intensity of the drugs were fairly high on Symmetry Shield$\mathrm{RP}_{18}$ column compared to other columns, with comparatively less peak tailing in VPL. Further optimization was made by the change in the aqueous/organic ratios of the mobile phase using the same column. Increase in the organic phase $(>70 \%)$ result in improper peak shapes, while ratio at $50: 50 \% \mathrm{~V} / \mathrm{V}$ the elution time was more than $4.0 \mathrm{~min}$. Mobile phase with $10 \mathrm{mmol}$ ammonium formate and ACN in a proportion of $30: 70 \% \mathrm{~V} / \mathrm{V}$ produce symmetric peaks, better retention and good peak response with less matrix effect.

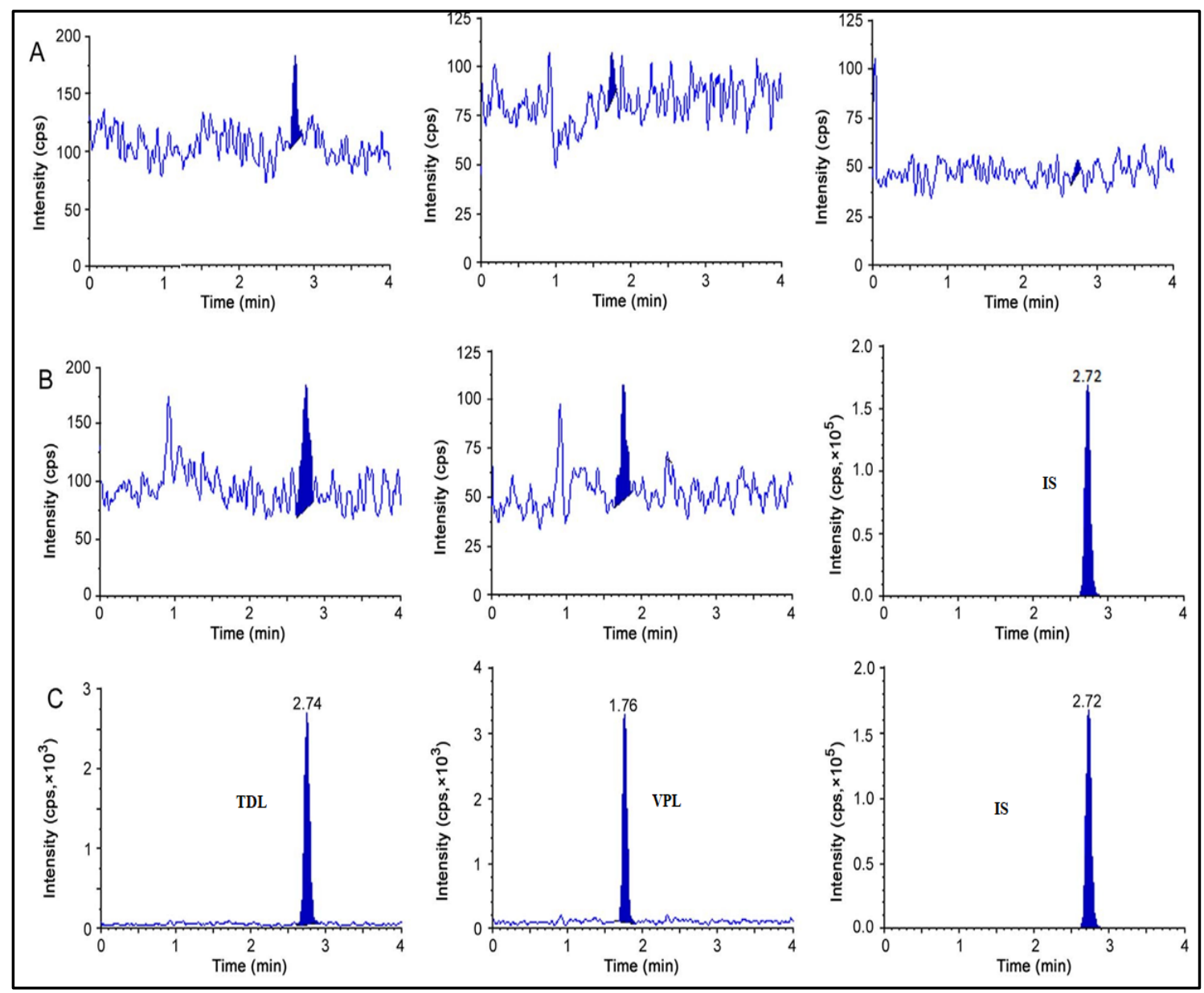

Fig. 3: Typical chromatograms of A) Double blank plasma B) Blank plasma with IS C) TDL, VPL at LLOQ level with IS

It was necessary to have the chromatographic analysis time of 4.0 min for higher sensitivity and better ionizatffiniency. The chromatograms in fig. 3A-C showed no interference with an elution time of TDL and VPL against endogenous components. However, as they eluted much ahead of VPL there was no interference in the quantitation and no further attempt was made towards their identification. The resolution factor between the analytes under the established conditions was 3.8, while the capacity factors for TDL and VPL were 2.01 and 0.93 , respectively. Further, there was no nosiness of medications at the elution time of the two drugs. Postcolumn injection chromatograms showed the lake of matrix effects with no signal improvement or suppression at TDL and VPL retention times (fig. 3 ).

Protein precipitation and liquid-liquid extraction methods were not providing precise and accurate results. As a result, Solid-phase extraction was processed on different separation cartridges, namely Bond Elut $\mathrm{C}_{18}$, Oasis-HLB, Phenomenex Strata-X, and Lichrosep-DVB. It was found that the addition of $2 \mathrm{mmol}$ ammonium formate buffer was essential during all stages of workup to obtain adequate response and consistency in the recovery with minimal matrix interference. The extracts found with Phenomenex cartridges were cleaner than remaining cartridges $[13,14]$.

\section{Method validation results}

The \% CV values for system suitability were obtained between $0.09 \%$ to $0.32 \%$ for the elution times and $0.52 \%$ to $1.57 \%$ for the peak response of two analytes and internal reference standard. For the determination of selectivity, the response for 10 different blank plasma lots at the elution times of analytes was equated with the peak response (at LLOQ). None of the blank plasma sources 
exhibited any obvious interference $(\leq 0.52 \%$ of LLOQ sample for TDL and VPL) at elution times of analytes.

The method was evaluated for carryover for column and autosampler with blank plasma after consequent infusion of ULOQ of analytes and the findings were $\leq 0.64 \%$. Peak response ratios (analyte/IS) against the sample concentrations were plotted to calculate linear regression. The linear curves were obtained over 5$1500 \mathrm{ng} / \mathrm{ml}$ range for TDL and $1-2000 \mathrm{ng} / \mathrm{ml}$ range for VPL. The corresponding regression equations for TDL and VPL were $\mathrm{y}=$
$(0.00089 \pm 0.00002) \quad x+(0.00052 \pm 0.00021) \quad$ and $y=(0.00025$ $\pm 0.00010) x+(0.00038 \pm 0.00008)$ respectively, with a correlation coefficient $\left(\mathrm{r}^{2}\right)$ of $\geq 0.9994$. For both the analytes, the bias of backcalculated values in calibration range was within $7.6 \%$ of the nominal concentration values. The signal-to-noise ratio at the LLOQ concentration was $\geq 15$ and $\geq 29$ for TDL and VPL respectively. The inter and intraday precision and accuracy findings were tabulated in table 1 . The relative standard deviations were $\leq 5.69 \%$ and the overall mean accuracy ranged from $93.00 \%$ to $104.00 \%$ for the two drugs.

Table 1: Trandolapril and verapamil data for intra and inter-day precision and accuracy

\begin{tabular}{|c|c|c|c|c|}
\hline \multirow[t]{2}{*}{ Nominal concentration $(\mathrm{ng} / \mathrm{ml})$} & \multicolumn{2}{|l|}{ Intra-day } & \multicolumn{2}{|l|}{ Inter-day } \\
\hline & Accuracy (\%) & \%RSD & Accuracy (\%) & \%RSD \\
\hline \multicolumn{5}{|l|}{ Trandolapril } \\
\hline 5 & 103.6 & 1.23 & 103.2 & 5.67 \\
\hline 10 & 93.2 & 4.9 & 97.1 & 5.2 \\
\hline 600 & 103.2 & 3.5 & 103.2 & 4.2 \\
\hline 1100 & 103.6 & 7.9 & 102.9 & 2.9 \\
\hline \multicolumn{5}{|l|}{ Verapamil } \\
\hline 1 & 104.5 & 5.2 & 107.5 & 5.1 \\
\hline 2 & 94.2 & 3.2 & 93.6 & 4.21 \\
\hline 600 & 98.2 & 4.8 & 94.6 & 5.1 \\
\hline 1500 & 95.9 & 0.59 & 95.1 & 0.92 \\
\hline
\end{tabular}

Table 2: Trandolapril and verapamil data for extraction recovery

\begin{tabular}{llll}
\hline Analyte and QC levels & peak response & $\begin{array}{l}\text { Extraction recovery } \\
\text { A/B (\%) }\end{array}$ \\
\cline { 2 - 3 } & $\begin{array}{l}\text { Pre-extraction } \\
\text { spiking (A) }\end{array}$ & $\begin{array}{l}\text { Post-extraction } \\
\text { spiking (B) }\end{array}$ & 97.33 \\
\hline Trandolapril & & & 37526 \\
LQC & 36525 & 365214 & 103.63 \\
MQC & 378455 & 10506345 & 95.23 \\
HQC & 10005236 & & 94.79 \\
Verapamil & & 37523 & 102.09 \\
LQC & 35568 & 532852 & 97.37 \\
MQC & 543985 & 5405892 & 97.83 \\
HQC & 5263521 & & 95.89 \\
Ledipasvir & & 945852 & 95.86 \\
LQC & 925345 & 975623 & \\
MQC & 935621 & 986521 \\
HQC & 945654 & & \\
\hline
\end{tabular}

Table 3: Matrix effect data for trandolapril and verapamil

\begin{tabular}{|c|c|c|c|c|c|c|}
\hline \multirow[t]{3}{*}{ Analytes/IS } & \multirow{2}{*}{\multicolumn{2}{|c|}{$\begin{array}{l}\text { Average area response }(n=6) \\
\text { Post-extraction spiking (A) }\end{array}$}} & \multirow{2}{*}{\multicolumn{2}{|c|}{ Neat samples in elution solution (B) }} & \multirow{2}{*}{\multicolumn{2}{|c|}{ Matrix factor (A/B) }} \\
\hline & & & & & & \\
\hline & LQC & HQC & LQC & HQC & LQC & HQC \\
\hline Trandolapril & 45615 & 13025468 & 46523 & 14086955 & 0.9804828 & 0.924648 \\
\hline Verapamil & 46750 & 6486531 & 48523 & 6542586 & 0.9634606 & 0.991432 \\
\hline Ledipasvir & 1045263 & 1203564 & 1123758 & 1305462 & 0.9301496 & 0.921945 \\
\hline
\end{tabular}

Table 4: Stability data for trandolapril and verapamil

\begin{tabular}{|c|c|c|c|c|c|}
\hline \multirow[t]{2}{*}{ Storage condition } & \multirow[t]{2}{*}{ QC level } & \multicolumn{2}{|c|}{ Accuracy (\%) } & \multicolumn{2}{|c|}{ Precision (\% CV) } \\
\hline & & TDL & VPL & TDL & VPL \\
\hline Bench-top stability & LQC & 35.14 & 98.23 & 1.25 & 2.01 \\
\hline$\left(8 \mathrm{~h}, 25^{\circ} \mathrm{C}\right)$ & HQC & 95.65 & 95.23 & 0.52 & 1.3 \\
\hline Freeze-thaw stability & LQC & 94.57 & 94.95 & 1.52 & 1.52 \\
\hline (at $-70^{\circ} \mathrm{C}, 5$ cycles) & $\mathrm{HQC}$ & 96.12 & 96.24 & 0.98 & 0.35 \\
\hline Auto-sampler stability & LQC & 94.21 & 97.84 & 0.58 & 0.84 \\
\hline$\left(74 \mathrm{~h}, 5^{\circ} \mathrm{C}\right)$ & HQC & 95.32 & 95.69 & 0.89 & 0.98 \\
\hline Processed sample & LQC & 94.68 & 98.23 & 0.68 & 0.74 \\
\hline stability $\left(16 \mathrm{~h}, 25^{\circ} \mathrm{C}\right)$ & $\mathrm{HQC}$ & 98.41 & 97.46 & 1.35 & 0.25 \\
\hline Long-term stability (at $-70^{\circ} \mathrm{C}$ for $116 \mathrm{~d}$,) & LQC & 97.25 & 96.58 & 1.25 & 0.26 \\
\hline & HQC & 97.66 & 97.32 & 0.36 & 1.25 \\
\hline
\end{tabular}


The average recoveries for TDL and VPL were found to be $92.9 \%$ and $93.5 \%$ respectively (table 2 ). The average recovery of the internal standard was $90.87 \%$. These findings specifies the developed technique was improbable to be modified by intra or inter-individual differences in the biomatrix and that the technique has good accuracy and reproducibility. The e ffects of matrixcomponents suppression or improvement, represented as IS normalized matrix effects, ranged from 0.965 to 1.245 for both the analytes (table 3 ). The \% CV findings for relative matrix ffect in lipemic, sodium-heparin plasma and haemolysed plasma lots also represents that no ion improvement or suppression of the analytes (intensities) (table 2).

The TDL and VPL stock solutions and ledipasvir were stable in methanol at refrigerated and room conditions for $2 \mathrm{w}$ and $24 \mathrm{~h}$ respectively. The bench top stability of analytes in plasma were proven up to $8 \mathrm{~h}$. Both the analytes were stable during 5 freeze-thaw cycles and for at least $74 \mathrm{~h}$ in the autosampler. Processed sample stability of the analytes was established up to $16 \mathrm{~h}$ at $25^{\circ} \mathrm{C}$ with no obvious difference in the concentration of TDL and VPL. The longterm stability (at-70 ${ }^{\circ} \mathrm{C}$ ) samples were stable for $116 \mathrm{~d}$ and findings were tabulated in table 4 .

Precision and accuracy results obtained in order to establish method ruggedness with dfferent RP-columns and different analysts were ranged from $0.83 \%$ to $4.25 \%$ and $97.60 \%$ to $98.37 \%$, respectively for TDL and VPL. Likewise, the \% CV and accuracy for dilution reliability of $1 / 2$ and $1 / 4$ th dilution varied from $1.67 \%$ to $3.27 \%$ and $95.6 \%$ to $99.25 \%$, respectively for two drugs.

\section{CONCLUSION}

An LC-MS/MS technique was developed for the sensitive and specific determination of trandolapril and verapamil in human plasma and validated using the ledipasvir internal reference standard. There was no interference or iffectixfrem endogenous substances in the quantitative analysis. The calibration range established for TDL and VPL was adequate using a small sample volume for the analysis. The optimized SPE protocol gave highly precise and consistent recovery for the two analytes with no further stages of dehydration and reconstitution.

\section{AUTHORS CONTRIBUTIONS}

All the author have contributed equally

\section{CONFLICT OF INTERESTS}

\section{Declared none}

\section{REFERENCES}

1. Berl T. Review: renal protection by inhibition of the reninangiotensin-aldosterone system. J Renin Angiotensin Aldosterone Syst 2009;10:1-8.

2. Diaz A, Ducharme A. Update on the use of trandolapril in the management of cardiovascular disorders. Vasc Health Risk Manag 2008;4:1147-58.

3. Guay DR. Trandolapril: a newer angiotensin-converting enzyme inhibitor. Clin Ther 2003;25:713-75.

4. Reynolds NA, Wagstaff AJ, Keam SJ. Trandolapril/verapamil sustained release: a review of its use in the treatment of essential hypertension. Drugs 2005;65:1893-914.

5. Tfelt-Hansen PC, Jensen RH. Management of a cluster headache. CNS Drugs 2012;26:571-80.

6. Merison K, Jacobs H. Diagnosis and treatment of a childhood migraine. Curr Treatment Options Neurol 2016;18:48.

7. Sreekanth N. HPTLC method development and validation of trandolapril in bulk and pharmaceutical dosage forms. J Adv Pharma Technol Res 2010;1:172-9.
8. Tapas Kumar Laha, Lopamudra Adhikari. RP-HPLC analysis of trandolapril in pharmaceutical dosage forms. Asian J Chem 2008;20:352-6.

9. Ganipisetty LA, Dachinamoorthy D. Stability indicating RPHPLC method development and validation of simultaneous estimation of trandolapril and verapamil hydrochloride with forced degradation studies in bulk and commercial products. Int J Pharma Res Scholars 2015;4:1-9.

10. Tejashwi E, Kiran Kumar S, Siddartha B. Simultaneous estimation and validation of Trandolapril and Verapamil in bulk and pharmaceutical dosage form by RP-HPLC method. World J Pharm Pharm Sci 2014;3:652-62.

11. Laxmi Madhuri P, Vusuvandla Geetha. Development and validation of RP-HPLC method for the simultaneous estimation of verapamil hydrochloride and Trandolapril in bulk and pharmaceutical dosage forms. Asian J Pharm Anal Med Chem 2016;4:38-46.

12. Senem Sanli, Nurullah Sanli. Development and validation of a liquid chromatographic method for the concurrent assay of weakly basic drug verapamil and amphoteric drug trandolapril in pharmaceutical formulations. J Food Drug Anal 2012;20:588-96.

13. Irene Panderi, Constantinos Pistos. Liquid chromatographic tandem mass spectrometric determination of trandolapril in human plasma. Anal Chim Acta 2005;540:375-82.

14. Naser Altannak, Ahmad Hemdan. UPLC-QToF-MS analysis of trandolapril and verapamil in the dosage form and spiked human plasma using solid phase extraction: stability indicating assay method. Curr Pharma Anal 2017;13:450-8.

15. US FDA, Guidance for industry bioanalytical method validation, food and drug administration, the center for drug evaluation and research (CDER), Rockville, Maryland, USA; 2001.

16. Anggun Aji Mukti, Fathul Jannah, Arief Nurrochmad, Endang Lukitaningsih. Development and validation method for quantitative determination of ciprofloxacin in human plasma and its application in bioequivalence test. Asian J Pharm Clin Res 2016;9:89-95.

17. Atul Vasanth D, Rajkamal B. A UPLC-MS/MS method development and validation for the estimation of pomalidomide from human plasma. Int J Appl Pharm 2017; 9:37-43.

18. International Conference on Harmonization (ICH) of Technical Requirements for the Registration of Pharmaceuticals for Human Use, Validation of analytical procedures: Methodology, ICH-Q2B, Geneva; 1996.

19. Validation of Analytical Procedures: Methodology. ICH Harmonised Tripartite Guidelines; 1995.

20. Marlina Ika, Rizka Andalusia, Supandi Supandi, Yahdiana Harahap. Simultaneous analytical method development of 6mercaptopurine and 6-methylmercaptopurine in dried blood spot using ultra performance liquid chromatography-tandem mass spectrometry. Int J Appl Pharm 2017;9:168-71.

21. Birva A Athavia, Zarna R Dedania, Ronak R Dedania, Vijayendra Swamy SM, Chetana B Prajapati. Stability indicating HPLC method for determination of vilazodone hydrochloride. Int J Curr Pharm Res 2017;9:123-9.

22. Wani TA. Ultra performance liquid chromatography-tandem mass spectrometric method development and validation for determination of neratinib in human plasma. South Afr J Chem 2015;68:113-25.

23. Brian Kiesel. LC-MS/MS assay for the quantitation of the tyrosine kinase inhibitor neratinib in human plasma. J Pharm Biomed Anal 2017;134:130-6.

24. Tijare LK, Rangari NT, Mahajan UN. A review on bioanalytical method development and validation. Asian J Pharm Clin Res 2016;9:6-10. 